\title{
Prolonged and biphasic acute hepatitis A in hepatitis B virus carrier
}

\author{
Elena Garlatti Costa, Michela Ghersetti, Silvia Grazioli, Pietro Casarin \\ Department of Internal Medicine, Santa Maria degli Angeli Hospital, Pordenone, Italy
}

\begin{abstract}
Acute hepatitis A is generally a self-limited disease in healthy subjects within few weeks, but an uncommon type of prolonged and biphasic acute course of hepatitis A infection has been also described. This type of presentation is observed in about 6-10\% of patients, but a small number of reports, concerning this topic, are available in literature. In addition, hepatitis A virus (HAV) infection in hepatitis B virus (HBV) carriers has rarely been discussed. A 41-year-old Italian man, already known to our Department for HBV infection as an inactive carrier HBsAg $(+)$ ve, experienced a prolonged and biphasic course of acute hepatitis A, lasting about 7 months. In this patient possible factors, causing the second flare of transaminases, were excluded (in particular autoimmunity). Liver biopsy as well HAV RNA search in blood/stools were not performed. In conclusion, the hepatologist should take into account this type of atypical course in patients with HAV-related hepatitis and should promote HAV vaccination in subjects with HBV-chronic hepatitis, to prevent possible life-threatening acute exacerbation of hepatic damage, mainly in $\mathrm{HBV}$-carriers with more severe forms of liver diseases.
\end{abstract}

\section{Introduction}

Acute hepatitis A may represent a possible pitfall for the hepatologist, because of its epidemiology and heterogeneous clinical presentation. Moreover, an increase in the prevalence of hepatitis A was recently re-

Correspondence: Elena Garlatti Costa, Department of Internal Medicine, Santa Maria degli Angeli Hospital, via Montereale 24,33170 Pordenone, Italy.

Tel.: +39.0434399280 - Fax: +39.0434399398.

E-mail: elena.garlatti@aas5.sanita.fvg.it

Key words: Hepatitis A virus; hepatitis B virus; inactive carrier $\mathrm{HBsAg}(+) v e$; prolonged and biphasic hepatitis A virus infection/relapsing hepatitis A; antibody.

Acknowledgments: the authors are grateful to Dr. Marina Crovatto and Dr. Endri Mauro for their helpful comments and suggestions.

Contributions: EGC, writing and drafting of manuscript, data collection and references search; MG, SG, writing of manuscript; PC, manuscript critical revising.

Conflict of interest: the authors declare no potential conflict of interest.

Received for publication: 28 December 2014.

Revision received: 28 June 2015.

Accepted for publication: 12 February 2016.

This work is licensed under a Creative Commons Attribution NonCommercial 4.0 License (CC BY-NC 4.0).

(C) Copyright E. Garlatti Costa et al., 2016

Licensee PAGEPress, Italy

Italian Journal of Medicine 2016; 10:241-244

doi:10.4081/itjm.2016.571 ported with an ongoing outbreak in Italy. ${ }^{1}$ Furthermore, hepatitis A infection, which is transmitted via an oral fecal route, can be asymptomatic, although some subjects can also present both jaundice and/or a fulminant course $(0.5-1 \%)$. About $70 \%$ of the adult population generally shows a symptomatic illness with nausea, vomiting, abdominal pain, headache and fever but only $30 \%$ of these patients develop jaundice. ${ }^{2} \mathrm{Ex}$ trahepatic manifestation of hepatitis A, such as cutaneous vasculitis and peripheral neuropathy, have been reported, although with a lower frequence. ${ }^{3}$ Then, in some situations acute hepatitis A acts as a trigger for the development of autoimmune hepatitis. ${ }^{4}$ On the basis of its clinical course, acute HAV infection is generally a self-limited disease, within few weeks (within 4-6 weeks), but in 6-10\% of cases hepatitis A course can be prolonged (more than 4 months), even though it is not defined as chronic. ${ }^{5-7}$ In the past, several case reports, concerning this atypical type of presentation, have been described. In particular, in 1992 Glikson et $a l .{ }^{8}$ reviewed their experience, concerning 14 cases of hepatitis A, showing a relapse, as well as 68 cases reported in the literature. They found that the relapse occurred at a rate, ranging between 3 and $20 \%$ of patients with acute hepatitis A, and it rarely showed a polyphasic course (multiple relapses). After a stage of typical hepatitis $\mathrm{A}$, a remission phase developed, with the partial or complete resolution of clinical and biochemical manifestations. Relapse usually occurred after a short period (usually less than 3 weeks) with a usually lower clinical severity than one, observed in the first phase, with variable liver function abnormalities and a tendency toward a more marked cholestatic feature. They concluded that the clinical course in patients, undergoing a relapse of hepatitis A infection, was almost al- 
ways benign and uneventful recovery is the rule with few exceptions. Steroid treatment, first reported in the past, resulted in marked clinical improvement and it was suggested that relapsing hepatitis A was associated with a persistent viremia as well as the presence of virus in stools during the relapse phase. The pathogenesis of prolonged hepatitis A probably involves an interaction between persistent viral infection and immune mechanisms responding to the persistent antigenic stimulation. Furthermore, there are few studies, concerning the natural history of hepatitis A in subjects known as inactive HBsAg $(+)$ ve carriers. Some of these Authors ${ }^{9}$ reported an increased risk of hepatitis A virus (HAV) superinfection with possible development of liver failure, in patients with a chronic hepatic damage, such as cirrhosis (especially among the elderly individuals), while other Authors ${ }^{10}$ observed that the decrease in hepatitis B virus (HBV) DNA, in patients with $\mathrm{HAV} / \mathrm{HBV}$ co-infection were not associated with a different clinical course in comparison with individuals without HBV infection. However, HAV vaccination, introduced in 1996, represents the best strategy to control the incidence of HAV infection and of its possible clinical complications (such as liver failure or the amount of fibrosis). In our paper, we report the case of a patient with chronic HBV-infection, undergoing an episode of acute and prolonged HAV-related hepatic injury.

\section{Case Report}

In the 2003, a 31-year-old Italian man was examined at our Department because of the first detection of HBsAg positivity. His past medical history was characterized by the following points: in 1982, when he was 10 years old, he experienced concussion and thoracic trauma after an accidental fall. He received a blood transfusion, during treatment because of multiple trauma, then when he was 18 years old he had viral meningitis with a self-limited course and no clinical consequences. Apart from the transfusion, his personal history was negative for risk factors for HBV infection and also for alcohol, smoke, drug intake and familiarity for chronic liver disease. He worked in a commercial firm and he was married with two sons in good health. Results of his blood test were the following: HBsAg(+)ve, $\mathrm{HBeAb}(+) v e, \mathrm{HBeAg}(-) \mathrm{ve}, \mathrm{HBcAb}$ $(+) v e, H B c A b$ immunoglobulin $\mathrm{M}$ (IgM) (-)ve, HBsAb (-)ve, HBV DNA $500 \mathrm{U} / \mathrm{mL}$, aspartate aminotransferase (AST) $23 \mathrm{U} / \mathrm{L}$, alanine aminotransferase (ALT) $33 \mathrm{U} / \mathrm{L}$, alkaline phosphatase (ALP) $71 \mathrm{U} / \mathrm{L}$, gamma-glutamyl transferase (GGT) $22 \mathrm{U} / \mathrm{L}$, total bilirubin $0.6 \mathrm{mg} / \mathrm{dL}$, normal platelets count, antibody profiles for hepatitis $\mathrm{C}$ and $\mathrm{D}$ virus and HIV were negative. On physical examination, neither hepatomegaly/splenomegaly nor chronic liver signs were detectable. An abdomen ultrasound (US) scan, excluded signs of chronic liver disease and/or portal hypertension while liver stiffness measurement (LSM) (with FibroScan) was equal to $3.8 \mathrm{kPa}$ [interquartile range (IQR) 0.8]. Later, the patient was followed in our Department. We concluded that the patient suffered from chronic HBV infection in the phase of inactive carrier HBsAg (+)ve. In August 2013, he was admitted to our medical Department because he experienced a symptomatic clinical course characterized by fever, malaise with headache, dyspepsia, vague abdominal pain and jaundice. He denied alcohol use or herbal medicine intake. He reported that one-week, before he had eaten uncooked fish. We planned a biochemical investigation, that showed: ALT $4300 \mathrm{U} / \mathrm{L}$, AST 2533 U/L, GGT 213 U/L, ALP 100 U/L, creatinin $0.75 \mathrm{mg} / \mathrm{dL}$, total bilirubin $7.0 \mathrm{mg} / \mathrm{dL}$, direct 5.2 $\mathrm{mg} / \mathrm{dL}$, albumin $2.9 \mathrm{~g} / \mathrm{dL}$, international normalized ratio $1.4 \mathrm{ammonium}, 81 \mathrm{mcmol} / \mathrm{L}$, white blood cells count $5680 / \mathrm{mmc}$, hemoglobin $14.9 \mathrm{~g} / \mathrm{dL}$, mean corpuscular volume $90 \mathrm{fL}$, platelets $231,000 / \mathrm{mmc}$, HAV IgM (+)ve, HBV DNA $684 \mathrm{U} / \mathrm{mL}$, ferritin 8051 $\mathrm{ng} / \mathrm{mL}$. In addition, blood markers of autoimmunity (mitochondrial antibody, smooth muscle antibody, antinuclear antibody, extractable nuclear antigen antibodies, anticytoplasmic autoantibodies, native DNA, liver kidney microsomal antibodies) as well as antigen and/or antibody profiles of the most important hepatotropic viruses (cytomegalovirus, Epstein-Barr virus, Herpes simplex virus -1 and -2) were negative. Major genetic mutations for hemochromatosis [C282Y and H63D] were excluded. Abdomen US scan showed signs of chronic hepatic disease with slight steatosis and associated fibrosis, gallbladder polypus $(4 \mathrm{~mm})$, reactive hilar node, splenomegaly (longitudinal diameter $12.5 \mathrm{~cm}$ ), normal caliber of portal vein. A case of acute hepatitis A in HBV chronic infection, was diagnosed and medical therapy with parenteral hydration was started, with an initial reduction in the hypertransaminasemia (Table 1) and a subsequent subjective improvement. So we discharged the patient from our hospital, but after one week he experienced a second peak of increased transaminases (Table 1) with asthenia and occasional headache. We repeated specific laboratory tests with the aim to exclude main causes of ALT flare, that were again negative with persistent IgM HAV Ab positive. Our patient was monitored with a bi-weekly biochemical profile (Table 1 and Figure 1). During the follow-up, the normalization of transaminases, negative IgM HAV antibodies and HBV DNA drop ( $<20 \mathrm{UI} / \mathrm{mL}$ on March 2014) were observed. We performed a second FibroScan that showed LSM values equal to $7.3 \mathrm{kPa}$ (IQR 1). Now the patient is well and continues with periodic clinical, laboratory and imaging follow-ups at the Hepatology Department of our Institution. 


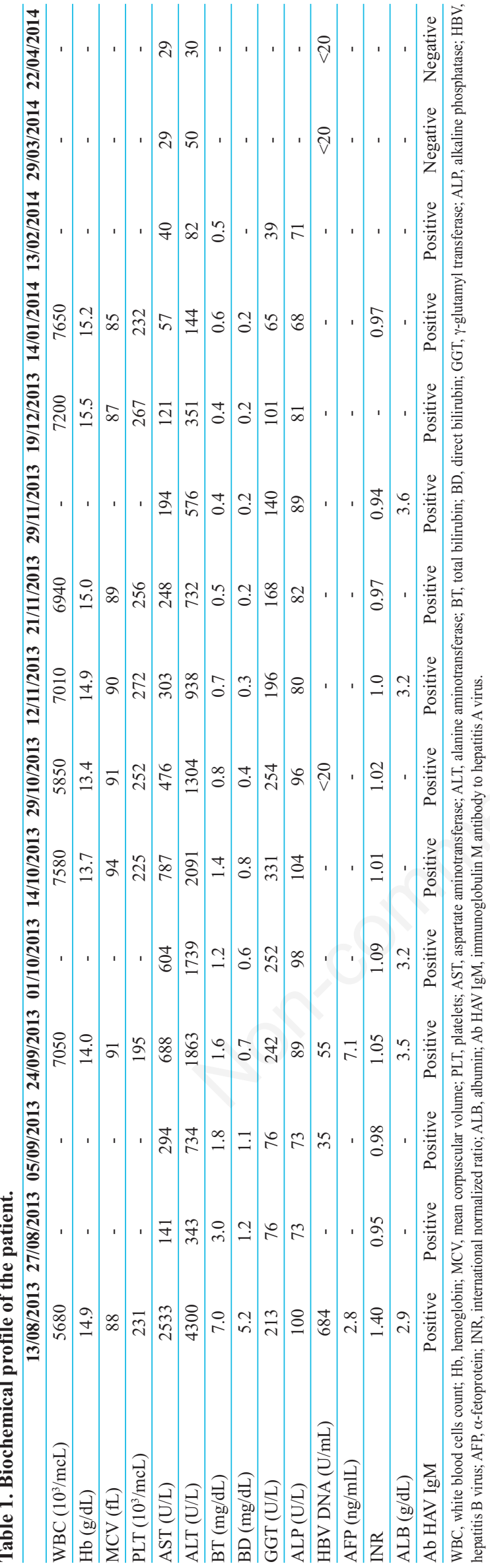

\section{Discussion and Conclusions}

We reported an uncommon type of HAV infection, characterized by a prolonged increase, lasting about 7 months, related to HAV infection with bimodal course, although it never reached the range of normality. Moreover, antibody profiles IgM HAV usually persist positive for about 13 weeks from the beginning of the infection, but the peak of transaminases often reaches a plateau after 4 weeks and decreases later and normalizes within 8 weeks. ${ }^{11}$ In prolonged HAV-infection, persistence of IgM anti-HAV antibodies (more than 13 weeks) is proportional to the persistence of circulating immune complexes and this is much more frequent in males. ${ }^{12}$ Although it was not possible to search HAV RNA in our hospital, we concluded that the second flare was attributable to persistent hepatitis A infection, after excluding the main causes of hypertransaminasemia (in particular autoimmunity and HBV flare). Moreover, it is interesting to note that IgM HAV absence was associated with transaminases reduction. On the basis of the presumed long-lasting HAV infection, a liver biopsy, which is an invasive examination, was not considered. Studies have shown that the excretion of Ag-HAV in stools approximately is associated with the persistence of ALT flares and this element is very important in considering the infectiousness of these subjects. ${ }^{13}$ We did not know why in some of these patients an infection with a prolonged course develops, but the immune response probably plays a major role, as suggested by the decrease in HBV DNA. In short, the suppression of hepatitis B virus replication is mediated by hepatitis A-induced cytokine production. In fact, the sharp rise in gamma-interferon production may be pivotal in the suppression of HBV replication in chronic HBV infection. ${ }^{14}$ The reasons for the different courses of hepatitis A infection in different patients remain unclear. We concluded, in accordance with different Authors, ${ }^{8}$ that the follow-up of these patients might be a sufficient tool, because the prognosis of HAV infection is usually benign, even if it relapses,

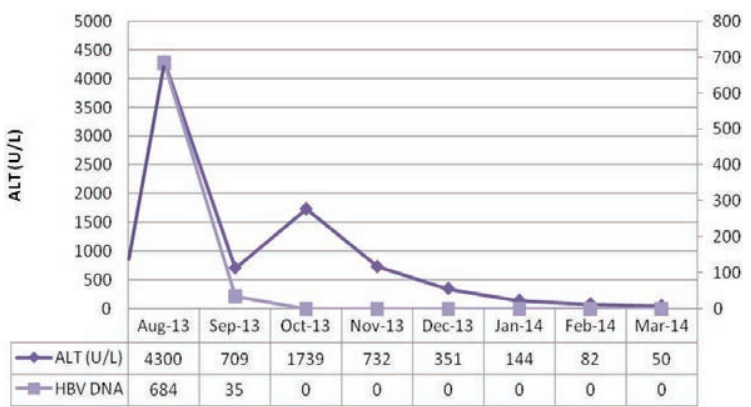

Figure 1. Biochemical profile of the patient. ALT, alanine aminotransferase; HBV, hepatitis $B$ virus. 
mainly in young patients with milder forms of liver injury, such as chronic hepatitis, whereas, elderly subjects with more serious forms of hepatic damage, such as cirrhosis, may be at higher risk of fulminant hepatitis and death. In conclusion, the hepatologist should take into account this type of atypical course in patients with HAV-related hepatitis and should promote HAV vaccination in subjects with HBV-chronic hepatitis, to prevent possible life-threatening acute exacerbation of hepatic damage, mainly in HBV-carriers with more severe forms of liver diseases.

\section{References}

1. Rizzo C, Alfonsi V, Bruni R, et al. Ongoing outbreak of hepatitis A in Italy: preliminary report as of 31 May 2013. Euro Surveill 2013;18:1-6.

2. Mauss S, Berg T, Rockstroh J. et al. Hepatology. A clinical textbook. Duesseldorf: Flying Publisher; 2009. pp 22-21.

3. Schiff ER. Atypical clinical manifestations of hepatitis A. Vaccine 1992;10:S18-20.

4. Tabak F, Ozdemir F, Tabak O, et al. Autoimmune hepatitis induced by prolonged hepatitis A virus infection. Ann Hepatol 2008;7:177-9.

5. Tanno H, Fay OH, Roman JA, Palazzi J. Biphasic form of hepatitis A virus infection: a frequent variant in Argentina. Liver 1988;8:53-7.

6. Verucchi G, Calza L, Chiodo F. Viral hepatitis A with atypical course. Clinical, biochemical, and virologic study of 7 cases. Ann Ital Med Int 1999;14:239-45.

7. Altman $\mathrm{C}$, Moliniè $\mathrm{C}$, Soudière $\mathrm{S}$, et al. Viral hepatitis A with prolonged course in adults. Gastroenterol Clin Biol 1996;20:42-6.

8. Glikson M, Galun E, Oren R, et al. Relapsing hepatitis A. Review of 14 cases and literature survey. Medicine (Baltimore) 1992;71:14-23.

9. Keeffe EB. Is hepatitis A more severe in patients with chronic hepatitis B and other chronic liver disease? Am J Gastroenterol 1995;90:201-5.

10. Sagnelli E, Coppola N, Pisaturo N, et al. Clinical and virological improvement of hepatitis $\mathrm{B}$ virus-related or hepatitis $\mathrm{C}$ virus-related chronic hepatitis with concomitant hepatitis A virus infection. Clin Infect Dis 2006;42:1536-43.

11. Koff RS. Clinical manifestations and diagnosis of hepatitis A virus infection. Vaccine 1992;10:S15-7.

12. Dautovic-Krkic S. Persistence of IgM anti HAV inprolonged form of HAV-infection. Med Arh 2005;59:91-3.

13. Dautovic-Krkic S, Cecuk D. Excretion of hepatitis A virus antigen in feces in acute and prolonged hepatitis A infection. Med Arh 2004;58:15-8.

14. van Nunen AB, Pontesilli O, Uytdehaag F, et al. Suppression of hepatitis $B$ virus replication mediated by hepatitis A-induced cytokine production. Liver 2001;21:45-9. 